\title{
Artificial neural networks (ANNs) in food science - A review
}

\author{
Sumit Goyal \\ Member, IDA, New Delhi, India \\ E-mail:thesumitgoyal@gmail.com
}

\begin{abstract}
Artificial Neural Networks (ANNs) have been applied in several areas, viz., science and technology, engineering, agriculture, life sciences and medicine as they have a remarkable ability to provide accurate results. This communication highlights the systematic information available in the literature concerning the implementation of ANN models for predicting properties of dairy products, fruits, vegetables and meat. The information presented in this review paper shows that over the last decade research related to ANN based predictive modelling in food science has picked up.
\end{abstract}

Keywords: artificial neural networks, artificial intelligence, soft computing, machine learning, dairy products, fruits, vegetables, meat products

\section{Introduction}

ANNs are inspired by the early models of sensory processing by the brain. An ANN can be created by simulating a network of model neurons in a computer. By applying algorithms that mimic the processes of real neurons, one can make the network 'learn' to solve many types of problems. A model neuron is referred to as a threshold unit. It receives input from a number of other units or external sources, weighs each input and adds them up. If the total input is above a threshold, the output of the unit is one; otherwise it is zero. Therefore, the output changes from 0 to 1 when the total weighted sum of inputs is equal to the threshold. The points in input space satisfying this condition define a so called hyperplane. In two dimensions, a hyperplane is a line, whereas in three dimensions, it is a normal plane. Points on one side of the hyperplane are classified as 0 and those on the other side as 1 . Thus, a classification problem can be solved by a threshold unit if the two classes can be separated by a hyperplane [1].

Shelf life studies provide important information to product manufacturers and developers to ensure that the consumer will continue to get a high quality product for a significant period of time after its production. Since the shelf life evaluation conducted in the laboratory is a long time-consuming process, and does not fit well with the speed requirements of the food industry, therefore accelerated methods for estimating the shelf life of food products have been recently innovated. The modern food industry has flourished because of its ability to deliver a wide variety of high quality food products to consumers. This has been achieved by building stability into the products through adopting various technological techniques like processing, packaging, and additives. Consumer demands for convenience have fueled new innovations in the food product development, packaging and chemical industries, and the widespread desire for products to use in the microwave oven has added further impetus to this effort. As an increasing number of new foods compete for getting space on supermarket shelves, the words "speed and innovation" have become the watchwords for food companies seeking to become "first to market" with successful products. Overall quality of the product is of prime importance in present day competitive market and needs to be built into the speed and innovation system. How the consumer feels about the product is the ultimate index of food quality. Therefore, the quality built in during the development of the product and production process must last through the distribution and consumption stages [2].

\section{ANNs in dairy products}

\subsection{Milk}

The usefulness of ANN models for prediction of shelf life of milk by multivariate interpretation of gas chromatographic profiles, and flavour-related shelf life was evaluated and compared with Principal Components Regression (PCR). The 
training set consisted of dynamic headspace gas chromatographic data collected during storage of pasteurized milk (input information for the ANN models used to make a decision) and its corresponding shelf life (prediction or response). The study revealed that ANN had better predictability than PCR. A standard error of the estimate of 2 days in shelf life resulting from regression analysis of experimental versus predicted values indicated a high predictability of ANN [3]. A methodology was developed for time series sales forecasting for short shelf life food products based on ANN models and evolutionary computing. The methodology was claimed to be particularly useful for manufacturers of fresh milk, since successful sales forecasting considerably reduces the lost sales and products returns [4].

\subsection{Yogurt}

\subsubsection{Control of expiration date}

The changes in the physical, chemical, and microbiological structure of yogurt determine the storage and shelf life of the product. In a reported study microbial counts and $\mathrm{pH}$ values of yogurt during storage were determined at day 1,7 , and 14. Simultaneously, image processing of yogurt was digitized by using a Machine Vision System (MVS) to determine colour changes during storage, and the obtained data were modeled with neurocomputing models for prediction of shelf life of set-type whole-fat and low-fat yogurts. The ANN models were developed using backpropagation networks with a single hidden layer and sigmoid activation functions [5]. The input variables of the network were $\mathrm{pH}$; total aerobic, yeast, mold, and coliform counts; and colour analysis values measured by the MVS. The output variable was the storage time of the yogurt. The modelling results showed that there was excellent agreement between the experimental data and predicted values, with a high determination coefficient $\left(R^{2}=0.9996\right)$ showing that the developed model was able to analyze non-linear multivariate data with very good performance, fewer parameters, and shorter calculation time. The model might be an alternative method to control the expiration date of yogurt shown in labeling and provide consumers with a safer food supply.

\subsubsection{Authenticity of low-fat yogurts}

The growing consumption of low- and reduced-fat dairy products demands routine control of their authenticity by health agencies. The usual analyses of fat in dairy products are very simple laboratory methods. However, they require manipulation and use of reagents of a corrosive nature, such as sulfuric acid, to break the chemical bonds between fat and proteins. Additionally, they generate chemical residues that require an appropriate disposal. The use of an ANN based on simple instrumental analyses, such as $\mathrm{pH}$, color, and hardness (inputs) was proposed for the classification of commercial yogurts in the low- and reduced-fat categories (outputs) [6]. A total of 108 strawberry-flavored yogurts (48 probiotic low-fat, 36 low-fat, and 24 full-fat yogurts) belonging to several commercial brands and from different batches were used in this research. The statistical analysis showed different features for each yogurt category. A database was built and a neural model was trained with the Levenberg-Marquardt algorithm by using the neural network toolbox of the software MATLAB 7.0.1. Validation with unseen data pairs showed that the proposed model was $100 \%$ efficient. Because the instrumental analyses do not require any sample preparation and do not produce any chemical residues, the proposed procedure is a fast and interesting approach for monitoring the authenticity of the products.

\subsubsection{Quantitative determination of protein}

A method has been introduced for quantitative determination of protein content in yogurt samples based on the characteristic absorbance of protein in 1800-1500 cm- 1 spectral region by mid-FTIR spectroscopy and chemometrics. Successive Projection Algorithm (SPA) wavelength selection procedure, coupled with feed forward Back-Propagation Artificial Neural Network (BP-ANN) model was the benefited chemometric technique. Relative Error of Prediction in BP-ANN and SPA-BP-ANN methods for training set was 7.25 and 3.70, respectively. Considering the complexity of the sample, the ANN model was found to be reliable, while the proposed method is rapid and simple, without any sample preparation step [7].

\subsection{Butter}

The seasonal variations of the fatty acids composition of butters were investigated over three seasons during a 12-month study in the protected designation of origin Parmigiano-Reggiano cheese area. Fatty acids were analyzed by GC-FID, and then computed by ANN [8]. Compared with spring and winter, butter manufactured from summer milk creams showed an optimal saturated/un-saturated fatty acids ratio $(-8.89$ and $-5.79 \%)$, lower levels of saturated fatty acids $(-2.63$ and $-1.68 \%)$ and higher levels of mono-unsaturated $(+5.50$ and $+3.45 \%)$, poly-unsaturated fatty acids $(+0.65$ and $+0.17 \%)$, and rumenic acid $(+0.55$ and $+3.41 \%)$, while vaccenic acid had lower levels in spring and higher in winter $(-2.94$ and $+2.91 \%)$. ANN models were able to predict the season of production of milk creams, and classify butters obtained from spring and summer milk creams on the basis of the type of feeding. 


\subsection{Swiss type cheeses}

$\mathrm{Ni}$ and Gunasekaran observed that a three-layer ANN model is able to predict more accurately than regression equations for the rheological properties of Swiss type cheeses on the basis of their composition [9]. Jimenez-Marquez et al. [10] were of the opinion that prediction of moisture in cheese of commercial production using neural networks models can be used both for research to develop the base of knowledge on production variables and their complex interactions, as well as for the prediction of cheese moisture.

\subsection{Processed Cheese}

Radial basis ANN and MLR computerized models were developed for determining the shelf life of processed cheese. Body and texture, aroma and flavour, moisture, and free fatty acids were taken as input variables, and sensory score as the output for developing the models. The results of experimental data were verified by comparing them with laboratory observations by employing mean square error, root mean square error, coefficient of determination and Nash-Sutcliffoe coefficient. Very good correlation was established between the experimental data and developed computerized models, thus establishing that computerized models are good for predicting the shelf life of processed cheese [11-13].

\subsection{Goat Milk Powder}

Goyal et al. [14] developed cascade multi layer models for analyzing the solubility index of roller dried goat whole milk powder. Input parameters were loose bulk density, packed bulk density, wettability and dispersibility, while solubility index was the output parameter. The results, like other reported studies [15-18], suggested that the developed models could provide an effective means of recognizing the patterns in data and accurately analyze the solubility index of roller dried goat whole milk powder

\subsection{Burfi}

Radial basis (exact fit) model was suggested for estimating the shelf life of extremely popular milk based sweetmeat namely burfi [19]. From the study it was concluded that the developed model, which is very convenient, less expensive and less time consuming can be a good alternative to expensive, time consuming and cumbersome laboratory testing method for estimating the shelf life of burfi [20-21].

\section{ANNs in fruits}

\subsection{Apple}

ANN modeling and several mathematical models were applied to predict the moisture ratio in an apple drying process by investigators. Four drying mathematical models were fitted to the data obtained from eight drying runs and the most accurate model was selected by researchers. Two sets of ANN modeling were also performed. In the first set, the data obtained from each pilot were modeled individually to compare the ANN predictions with the best mathematical model. In the second set of ANN modelling, the simultaneous effect of all the four input parameters including air velocity, air temperature, the thickness of apple slices and drying time was investigated. The results showed that the ANN predictions were more accurate in comparison with the best fitted mathematical model. In addition, none of the mathematical models was able to predict the effect of the four input parameters simultaneously, while the presented ANN model predicted this effect with a good precision [22].

\subsection{Apple juice}

Freeze-drying of foamed and non foamed apple juice was studied by Raharitsifa and Ratti [23] in order to assess if there is a reduction in process time due to foaming. Foams were prepared by whipping apple juice with methylcellulose or egg albumin at different concentrations. Foamed and non foamed juice samples having different thickness and different initial weight were frozen at $-40^{\circ} \mathrm{C}$, and then freeze dried at $20^{\circ} \mathrm{C}$ during $48 \mathrm{~h}$ under vacuum. Sample weight loss and temperature were followed at different process times. A mathematical model based on ANN was developed to represent foam kinetics and temperature curves during freeze-drying. This study revealed that freeze-drying of foamed materials is limited by heat transfer, while for non foamed ones, by mass transfer. It was shown that the insulation property characteristic of foams was more significant in slowing down the freeze-drying process than the increased surface area 
available for mass transfer due to foaming. ANN can be used to obtain excellent predictions of moisture content and temperature during the freeze-drying process.

\subsection{Banana}

An electronic nose based system, which employs an array of inexpensive commercial tin-oxide odour sensors, was developed by Llobet et al. [24] to analyze the state of ripeness of bananas. Readings were taken from the headspace of three sets of bananas during ripening over a period of 8-14 days. A principal components analysis (PCA) and investigatory techniques were used to define seven distinct regions in multisensor space according to the state of ripeness of the bananas, predicted from a classification of banana-skin colours. Then three supervised classifiers, namely Fuzzy ARTMAP, LVQ and MLP were used to classify the samples into the observed seven states of ripeness. It was found that the Fuzzy ARTMAP and LVQ classifiers outperformed the MLP classifier, with accuracies of $90.3 \%$ and $92 \%$, respectively, compared to MLP classifier (83.4\%). Furthermore, these methods were able to predict accurately the state of ripeness of unknown sets of bananas with almost the same accuracy, i.e., 90\%. Finally, workers showed that the Fuzzy ARTMAP classifier, unlike LVQ and MLP, is able to perform efficiently on-line learning in this application without forgetting previously learnt knowledge. All of these characteristics make the Fuzzy-ARTMAPbased electronic nose a very attractive instrument with which to determine non-destructively the state of ripeness of fruit.

\subsection{Cherries}

A machine vision system was created to identify different types of tissue characteristics on cherries. It consisted of an enhanced NIR range vidicon black and white camera (sensing range 400-2000 nm), a monochrometer controlled light source and a computer. Multiple spectral images of cherry samples were collected over the 680-1280 nm range at increments of $40 \mathrm{~nm}$. Using the spectral signatures of different tissues on cherry images, ANN modelling was implemented to pixel-wise classification. An enhanced genetic algorithm was applied to design the topology and evolve the weights for multilayer feedforward ANN. An average of $73 \%$ classification accuracy was achieved for correct identification as well as quantification of all types of cherry defects [25].

\subsection{Grape}

Janik et al. [26] conducted a study to compare the performance of partial least squares (PLS) regression analysis and ANN for the prediction of total anthocyanin concentration in red-grape homogenates from their visible-near-infrared (Vis-NIR) spectra. The PLS prediction of anthocyanin concentrations for new-season samples from Vis-NIR spectra was characterized by regression non-linearity and prediction bias. In practice, this usually requires the inclusion of some samples from the new vintage to improve the prediction. The use of WinISI LOCAL partly alleviated these problems but still resulted in increased error at high and low extremes of the anthocyanin concentration range. ANN regression was investigated as an alternative method to PLS, due to the inherent advantages of ANN for modelling non-linear systems. The proposed method combines the advantages of the data reduction capabilities of PLS regression with the non-linear modelling capabilities of ANN. With the use of PLS scores as inputs for ANN regression, the model was shown to be quicker and easier to train than using raw full-spectrum data. The ANN calibration for prediction of new vintage grape data, using PLS scores as inputs, was more linear and accurate than global and LOCAL PLS models and appeared to reduce the need for refreshing the calibration with new-season samples. ANN with PLS scores required fewer inputs and was less prone to overfitting than using PCA scores. Researchers concluded that the variation of ANN method, using carefully selected spectral frequencies as inputs, resulted in prediction accuracy comparable to those using PLS scores but, as for PCA inputs, was also prone to overfitting with redundant wavelengths.

\subsection{Guava}

The respiration rate prediction of fresh produce is crucial for designing and operating postharvest storage systems. The investigators constructed and evaluated respiration models of guava fruit by using not only the enzyme, chemical kinetics but also ANN with the experimental data obtained from 5, 10, 15, 20, 25 and $30{ }^{\circ} \mathrm{C}$ (for constructing) as well as 12 and $22{ }^{\circ} \mathrm{C}$ (for evaluating) by closed system method. All the developed models showed good agreement with actual observations. As regards fidelity the ANN model with topologic structure of $3 \times 9 \times 1$ trained by the LevenbergMarquardt algorithm, evaluation results were such that the mean absolute percentage error (MAPE) and the two-tailed Pearson correlation coefficient (r) were 5.31 and 0.997 for $12{ }^{\circ} \mathrm{C}, 4.85$ and 0.995 for $22{ }^{\circ} \mathrm{C}$, had superiority over the two other models. The results indicated that the ANN approach is a more precise method, and can be used for predicting the respiration rate of guava fruit [27]. 


\subsection{Orange}

ANNs are among the most popular techniques for nonlinear multivariate calibration in complicated mixtures using spectrophotometric data. In this study, Fe and $\mathrm{Ni}$ were simultaneously determined in aqueous medium with xylenol orange $(\mathrm{XO})$ at $\mathrm{pH}$ 4.0. In this way, after reducing the number of spectral data using PCA, an ANN consisting of three layers of nodes was trained by applying a backpropagation learning rule. Sigmoid transfer functions were used in the hidden and output layers to facilitate nonlinear calibration. The ANN method gave satisfactory results [28].

\subsection{Pineapple}

Boonmung et al. [29] planned a study for evaluating performance of ANN modelling for pineapple grading. The main objective of their experiment was to evaluate resonant frequency, firmness and soluble solids for pineapple classification using ANN as the analytical tool. A sample of 149 pineapples was classified based on their internal qualities into five classes: unripe, partially ripe, ripe, partially overripe and completely overripe. The proposed ANN model successfully classified pineapples into merely three classes as unripe, ripe and completely overripe. The most effective model was obtained when both resonant frequency and soluble solids were included in the model. The classification accuracy was more than $83 \%$ for all three classes.

\subsection{Pomegranate}

Drying of pomegranate arils was predicted by Motevali et al. [30] by creating ANN and mathematical models. Ten semi-theoretical and empirical models were fitted to the experimental data to evaluate and select the best model for thin-layer drying of pomegranate arils. Experiments were conducted at six temperature levels of 45, 50, 55, 60, 65 and $70{ }^{\circ} \mathrm{C}$, and three levels of air velocity $(0.5,1$ and $1.5 \mathrm{~m} / \mathrm{s})$. Microwave pre-treatments were used for samples and the results were compared with those of control (no pre-treatments). Regression analysis of mathematical models showed that the Midili model fitted best to the measured data. However, regarding $\mathrm{R}^{2}$ and MSE criteria, ANN modelling yielded a better prediction of pomegranate arils moisture ratio during drying of arils compared to all the mathematical models studied.

\subsection{Strawberry}

In order to provide an efficient and running analytical tool to strawberry plant breeders who have to characterize and compare the aromatic properties of new cultivars to those already known, a HS-SPME/GC-MS analysis method has been coupled with a statistical treatment method issued from the current development of ANN, and more specifically, the unsupervised learning systems called Kohonen self-organizing maps (SOMs). 70 strawberry samples harvested at CIREF from 17 known varieties were extracted by using a DVB/Carboxen/PDMS SPME fiber according to the headspace procedure, and then chromatographed. Panels of 23 characteristic aromatic constituents were selected according to the published results relative to strawberry aroma. The complex resulting matrix, collecting the relative abundance of the 23 selected constituents for each sample were used as inputs, into the SOM software adapted and optimized from the Kohonen approach described by one of the authors. After a period of training, the self-organized system afforded a map of virtual strawberries to which real samples were compared and plotted in the best matching unit (BMU) of the map. The efficiency for discriminating the real samples according to their variety is dependent on the number of units selected to define the map. In this case, a 24-unit map allowed the complete discrimination of the 17 selected varieties. Moreover, to test the validity of this approach, two additional samples were blind-analyzed and the results were computed according to the same procedure. At the end of this treatment, both samples were plotted into the same unit as those of the same variety used for training the map. Results from ANN modelling were found to be satisfactory [31].

\section{ANN in Vegetables}

\subsection{Beans}

A computer vision system (CVS) was created for the quality inspection of beans, based on size and color quantification of samples. The system consisted of hardware and software. The hardware was created to capture a standard image from the samples. The software was coded in MATLAB for segmentation, morphological operation and color quantification of the samples. For practical application of the software, a user-friendly interface was designed using MATLAB graphical user interface (GUI). Length and width of the samples were determined using this system. Then 
the results of the system were compared to the measurements obtained by a caliper. High correlations $(r=0.984$ and 0.971 for length and width, respectively) were obtained between the results of the system and the caliper measurements. Moment analysis was performed to identify the beans based on their intensity distribution. Average, variance, skewness and kurtosis values were determined for each channel of red, green, blue (RGB) color format. ANNs were used for color quantification of the samples. Samples classified by human inspectors into five classes and twelve moment values of the 69 samples with their classes were used in the training stage of ANN. Testing of the ANN was performed with other 371 samples. The automated system was able to correctly classify $99.3 \%$ of white beans, $93.3 \%$ of yellow-green damaged beans, $69.1 \%$ of black damaged beans, $74.5 \%$ of low damaged beans and $93.8 \%$ of highly damaged beans. The overall correct classification rate obtained was $90.6 \%$ confirming the significance of ANN modelling [32].

\subsection{Broccoli}

Broccoli grading was studied based on computer vision and ANN. Broccoli images were captured and the five parameters of color and shape (b*, TCD, Hdeg, proportion of yellowness area, roundness) were extracted and calculated from those images by images analysis method such as background purification, color segmentation, gray transform etc. A new grading method was provided based on the results of image analysis. The five parameters used as input vector to establish the Backpropagation Neural Network (BNN) for improving prediction precision, and another four ANNs Probabilistic Neural Network, Self-Organizing Competition Neural Network, Learning Vector Quantization Neural Network and Self-Organizing Feature Map Neural Network were also used as classifier in MATLAB 7.0. The results showed that all the five neural networks could be used for broccoli grading with the forecasting accuracy at the range of 68.2 93.4\%. The BNN was the best network with the forecasting accuracy of $93.4 \%$ [33].

\subsection{Cabbage}

ANNs are universal and flexible models for linear and non-linear systems. A study was conducted to modeling an ecosystem using ANN models and the conventional model, and assessing their effectiveness in the dynamic simulation of ecosystem. Elman ANN model, linear ANN model, and linear ordinary differential equation were developed to simulate the dynamics of Chinese cabbage growth system recorded in the field. MATLAB codes for these ANN models were given. Sensitivity analysis was conducted to detect the robustness of these models. The results showed that Elman ANN model could simulate the multivariate non-linear system at the desired accuracy, indicating that sensitivity analysis is powerful to detect the robustness and stability of neural network models [34].

\subsection{Carrot}

Erenturk and Erenturk [35] analyzed drying kinetic of carrot considering different drying conditions. The drying experiments were performed at four levels of drying air temperatures of $60-90{ }^{\circ} \mathrm{C}$, together with three levels of air flow velocities of $0.5-1.5 \mathrm{~m} / \mathrm{s}$, and also three levels of thickness $0.5-1 \mathrm{~cm}$. Four different MMs available in the literature were fitted to the experimental data by researchers. Among the considered mathematical drying models, modified Page model was found to be more suitable for predicting the drying of carrot. In order to optimize MMs obtained by using regression analysis, genetic algorithm was used. In all stages of the mathematical modeling, genetic algorithms were applied. In addition, a FNN was employed to estimate moisture content of carrot. Backpropagation algorithm, the most common learning method for the FNN, was used in training and testing the ANN. Comparing the $r$ (correlation coefficient), $r^{2}$ (coefficient of determination), $\chi^{2}$, and sum of squares of the difference between the experimental data and fit values of the four models, together with the optimized model by using genetic algorithms and the FNN based estimator, it was concluded that the FNN represented drying characteristics of carrot better than the others.

\subsection{Ginger}

Curcuma amada (Mango ginger) was dried at four different power levels ranging 315-800 W to determine the effect of microwave power on moisture content, moisture ratio, drying rate, drying time and effective diffusivity. A FNN using backpropagation algorithm was employed to predict the moisture content during microwave drying. It was found to be quite adequate for predicting the drying kinetics with $R^{2}$ of 0.985 [36].

\subsection{Onion}

Melin et al. [37] performed experiments on real prices of green onion and tomato in the U.S. using modular ANN with fuzzy integration applied for time series forecasting. They implemented several ANN architectures to the problem of simulating and predicting the dynamic behaviour of complex economic time series. They also compared the simulation 
results with the traditional statistical model. The results suggested that real prices of green onion and tomato in the U.S. show complex fluctuations in time and are very complicated to predict with traditional statistical approaches.

\subsection{Spinach}

An attempt was made to retrieve the spinach crop parameters like biomass, leaf area index, average plant height and soil moisture content by using the X-band scattering coefficients with backpropagation ANN at different growth stages of this crop. The research confirmed the utility of backpropagation ANN in handling such a non-linear dataset. Different transfer functions, e.g., tansig, logsig and purelin were used and the performance of the ANN was optimized by changing the number of neurons in the hidden layers. The study suggested the need of critical analysis of the backpropagation ANN in terms of selection of the best transfer function and other network parameters for better results [38].

\section{$5 \quad$ ANN in meat products}

\subsection{Chicken}

In the areas where broiler industry is located, poultry manure from chicken farms could be a major source of ground water pollution, and this may have extensive effects particularly when the farms use nearby ground water as their fresh water supply. Karadurmus et al. [39] investigated modelling of the effects of chicken manure on ground water by using ANNs. An ANN model was developed to predict the total coliform in the ground water well in poultry farms. The BNN algorithm was employed for training and testing the network, and the Levenberg-Marquardt (LM) algorithm was utilized for optimization. The MATLAB 7.0 environment with Neural Network Toolbox was used for coding. Given the associated input parameters such as the number of chickens, type of manure pool management and depth of well, the model estimated the possible amount of total coliform in the wells to a satisfactory degree, which is expected to be of help in future for estimating the ground water pollution resulting from chicken farms.

\subsection{Fish}

In a similar study, Hosseini et al. [40] presented intelligent processing of information collected by a portable 32-sensor electronic nose for fish freshness assessment. They used ANNs to design an intelligent system for measuring the freshness of spoiling fish. Investigators found that the degree of selectivity and the type of odours that could be detected by the electronic nose are largely dependent on the choice and number of sensors in the sensor array. They identified the most sensitive sensors, which provide a good estimation of freshness assessment in a laboratory environment. The information processing method was applied to collected data from four species. The procedure was repeated every day during a period often days after catching the fish. It was observed that the proposed intelligent system can successfully identify the number of days after catching the fish with an accuracy of up to of $91 \%$, thus the proposed assessment method can be used in designing an intelligent fish freshness assessment system.

\subsection{Hen}

ANN is capable of modelling any complex function and can be used in poultry and animal production areas. Savegnago et al. [41] investigated the possibility of using ANN on an egg production dataset and fitting models to the egg production curve by applying 2 approaches, one using a nonlinear logistic model and the other using 2 ANN models, viz., MLP and RBFNN. Two datasets from 2 generations of a White Leghorn strain that had been selected mainly for egg production were used. In the first dataset, the mean weekly egg-laying rate was ascertained over a 54-wk egg production period. This dataset was used to adjust and test the logistic model and to train and test the ANNs. The second dataset covering $52 \mathrm{wk}$ of egg production was used to validate the models. The mean absolute deviation (MAE), MSE, and $\mathrm{R}^{2}$ were used to evaluate the fit of the models. The MLP neural network had the best fit in the test and validation phases. The advantage of using ANNs is that they can be fitted to any kind of dataset and do not require model assumptions such as those required in the nonlinear methodology. The results confirmed that MLP neural networks can be used as an alternative tool to fit to egg production. The benefits of the MLP are the great flexibility and lack of prior assumptions, when estimating a noisy nonlinear model. 


\subsection{Lamb}

Peiyuan and Man [42] analyzed and established multiple data fusion model with ANN training for testing freshness of lamb meat. Digital image processing techniques were used to detect smell and $\mathrm{pH}$ value as well as surface colour quality of lamb and other information.

\subsection{Pork}

Valous et al. [43] used a small portion of uncorrelated singular values as robust features for the classification of sliced pork ham images using a supervised ANN classifier. Images were acquired from four qualities of sliced cooked pork ham typically consumed in Ireland (90 slices per quality) having similar appearances. Mahalanobis distances and Pearson product moment correlations were used for feature selection. Six highly discriminating features were used as input to train the ANN. An adaptive FANN classifier was employed to obtain a suitable mapping from the input dataset. The overall correct classification performance for the training, validation and test set were $90.3 \%, 94.4 \%$, and $86.1 \%$, respectively. The results confirmed that the classification performance was satisfactory. Extracting the most informative features led to the recognition of a set of different but visually quite similar textural patterns based on quaternionic singular values.

\subsection{Sausage}

An ANN model was developed to predict the temperature and moisture content of sausage (frankfurters) during smokehouse cooking [44]. Fat protein ratio (FP), initial moisture content, initial temperature, radius of frankfurter, ambient temperature, relative humidity and process time were input variables. Temperature at the frankfurter centre, average temperature of the frankfurter and average moisture content (d.b) of the frankfurter were outputs. Network training data were obtained from validated heat and mass transfer models simulating temperature and moisture profiles of a frankfurter. Backpropagation method was used for ANN training. Selection of hidden nodes, learning rate, momentum and range of input variables were important to ANN prediction. The developed model efficiently predicted the temperature and moisture content of sausage (frankfurters) during smokehouse cooking.

\subsection{Turkey}

ANN is a relatively new option to model growth in animal production systems. One self-organizing sub model of ANN is the group method of data handling (GMDH)-type ANN. The use of such self-organizing networks has led to successful application of the GMDH algorithm over a broad range of areas in engineering, science, and economics. Mottaghitalab et al. [45] aimed to apply the GMDH-type ANN to predict caloric efficiency (CE, g of gain/kcal of caloric intake) and feed efficiency (FE, $\mathrm{kg}$ of gain/kg of feed intake) in tom and hen turkeys fed diets containing different energy and amino acid levels. Involved effective input parameters in prediction of CE and FE were age, dietary ME, CP, Met, and Lys. Quantitative examination of the goodness of fit for the predictive models was made using $\mathrm{R}^{2}$ and error measurement indices commonly used to evaluate forecasting models. Statistical performance of the developed GMDH-type ANN models revealed close agreement between observed and predicted values of CE and FE. Researchers concluded that using such powerful models can enhance ability to predict economic traits, make precise prediction of nutrition requirements, and achieve optimal performance in poultry production.

\section{Conclusion}

The extensive review presented in this paper shows the effectiveness of ANN in food science. It is observed that in last decade research related to ANN predictive modelling has picked up. This review shows that ANN has been a successful tool in predicting food properties of dairy products, fruits, vegetables and meat products. ANN, as compared to laboratory testing provides a low cost and fast method for prediction in food science area. Also, ANNs have proved to be accurate for predictive analysis of food products.

\section{References}

[1] Krogh, A. (2008). What are artificial neural networks? Nature Biotechnology, 26(2), 195-197.

[2] www.medlabs.com/Downloads/food_product_shelf_life_web.pdf (accessed on 20.7.2013).

[3] Vallejo-Cordoba, B., Arteaga, G. E and Nakai, S. (1995). Predicting milk shelf-life based on artificial neural networks and headspace gas chromatographic data. Journal of Food Science, 60, 885-888. 
[4] Doganis, P., Alexandridis, A., Patrinos, P. and Sarimveis, H. (2006). Time series sales forecasting for short shelf-life food products based on artificial neural network models and evolutionary computing. Journal of Food Engineering, 75,196-204.

[5] Sofu, A., and Ekinci, F.Y. (2007). Estimation of Storage Time of Yogurt with Artificial Neural Network Modeling. Journal of Dairy Science, 90(7), 3118-3125.

[6] Cruz. A.G., Walter, E.H.M., Cadena, R.S., Faria, J.A.F., Bolini, H.M.A., and Fileti, A.M.F. (2009). Monitoring the authenticity of low-fat yogurts by an artificial neural network. Journal of Dairy Science, 92(10), 4797-4804.

[7] Khanmohammadi, M., Garmarudi, A.B., Ghasemi, K., Garrigues, S., and Guardia, M. (2009). Artificial neural network for quantitative determination of total protein in yogurt by infrared spectrometry. Microchemical Journal, 91 (1), 47-52.

[8] Gori, A., Chiara, C., Selenia, M., Nocetti, M., Fabbri, A., Caboni, M. F., and Losi, G. (2011). Prediction of seasonal variation of butters by computing the fatty acids composition with artificial neural networks. European Journal of Lipid Science and Technology, 113(11), 14121419 .

[9] Ni, H., and Gunasekaran, S. (1998). Food quality predication with neural networks. Food Technology, 52 (10), 60-65.

[10] Jimenez-Marquez, S. A., Thibault, J., and Lacroix, C. (2005). Prediction of moisture in cheese of commercial production using neurocomputing models. International Dairy Journal, 15, 1156-1174.

[11] Goyal, Sumit, and Goyal, G.K. (2013). Intelligent artificial neural network computing models for predicting shelf life of processed cheese. Intelligent Decision Technologies, 7(2), 107-111.

[12] Goyal, G.K., and Goyal, Sumit (2013). Cascade artificial neural network models for predicting shelf life of processed cheese. Journal of Advances in Information Technology, 4(2), 80-83.

[13] Goyal, Sumit, and Goyal, G.K. (2012).Application of simulated neural networks as non-linear modular modeling method for predicting shelf life of processed cheese. Jurnal Intelek, 7(2), 48-54.

[14] Goyal, Sumit, Sitanshu, K., and Goyal, G.K. (2013).Artificial neural networks for analyzing solubility index of roller dried goat whole milk powder. International Journal of Mechanical Engineering and Computer Applications, 1(1), 1-4.

[15] Goyal, Sumit, and Goyal, G.K. (2013) Radial basis artificial neural network models for predicting solubility index of roller dried goat whole milk powder. In: V. Snášel et al. eds. Soft Computing in Industrial Applications. Advances in Intelligent Systems and Computing 223, DOI: 10.1007/978-3-319-00930-8_21. Chapter No.: 21, Book ID: 311964_1_En Book. ISBN: 978-3-319-00929-2. Publisher: Springer International Publishing, Switzerland.

[16] Goyal, Sumit, and Goyal, G.K. (2012).Predicting solubility index of roller dried goat whole milk powder using Bayesian regularization ANN models. Scientific Journal of Zoology, 1(3), 61-68.

[17] Goyal, Sumit, and Goyal, G.K. (2012). Multilayer ANN models for determining solubility index of roller dried goat whole milk powder. Journal of Bioinformatics and Intelligent Control, 1, 76-79.

[18] Goyal, Sumit, and Goyal, G.K. (2013).Cascade modelling for predicting solubility index of roller dried goat whole milk powder. Bulletin of Electrical Engineering and Informatics, 2(1).

[19] Boishebert, V.D., Urruty, L., Giraudel, J.L., and Montury, M. (2004). Assessment of strawberry aroma through solid-phase microextraction-gas chromatography and artificial neuron network methods. Variety classification versus growing years. Journal of Agricultural and Food Chemistry, 52(9), 2472-2478.

[20] Goyal, Sumit, and Goyal, G.K. (2012). Radial basis (exact fit) artificial neural network technique for estimating shelf life of burfi. Advances in Computer Science and its Applications, 1(2), 93-96.

[21] Goyal, Sumit, and Goyal, G.K. (2013). Artificial vision for estimating shelf life of burfi. Journal of Nutritional Ecology and Food Research, 1(2), 1-3.

[22] Khoshhal, A., Dakhel, A.A., Etemadi, A., and Zereshki, S. (2010). Artificial neural network modeling of apple drying process. Journal of Food Engineering, 33, 298-313.

[23] Raharitsifa, N., and Ratti, C. (2010). Foam-mat freeze-drying of apple juice part 1: Experimental data and ANN simulations. Journal of Food Process Engineering, 33, 268-283.

[24] Llobet, E., Hines, E.L., Gardner, J.W., and Franco, S. (1999). Non-destructive banana ripeness determination using a neural network-based electronic nose. Measurement Science and Technology, 10(6), 538-548.

[25] Guyer, D., and Yang, X. (2000). Use of genetic artificial neural networks and spectral imaging for defect detection on cherries. Computers and Electronics in Agriculture, 29(3), 179-194.

[26] Janik, L.J., Cozzolino, D., Dambergs, R., Cynkar, W., and Gishen, M. (2007). The prediction of total anthocyanin concentration in red-grape homogenates using visible-near-infrared spectroscopy and artificial neural networks. Analytica Chimica Acta, 594(1), 107-118.

[27] Wang, Z., Duan, H., and Hu, C. (2009). Modelling the respiration rate of guava (Psidium guajava L.) fruit using enzyme kinetics, chemical kinetics and artificial neural network. European Food Research and Technology, 229(3), 495-503.

[28] Kompany-Zareh, M., Massoumi, A., and Pezeshk-Zadeh, S. (1999). Simultaneous spectrophotometric determination of Fe and Ni with xylenol orange using principal component analysis and artificial neural networks in some industrial samples. Talanta, 48(2), $283-292$.

[29] Boonmung, S., Chomtee, B., and Kanlayasiri, K. (2006). Evaluation of artificial neural networks for pineapple grading. Journal of Texture Studies, 37(5), 568-579.

[30] Motevali, A., Minaei, S., Khoshtaghaza, M.H., Kazemi, M., and Nikbakht, A. M. (2010). Drying of pomegranate arils: comparison of predictions from mathematical models and neural networks. International Journal of Food Engineering, 6(3), 1-20.

[31] Urruty, L., Giraudel, J.L., Lek, S., Roudeillac, P., and Montury, M. (2002). Assessment of strawberry aroma through SPME/GC and ANN methods, classification and discrimination of varieties. Journal of Agricultural and Food Chemistry, 50 (11), 3129-3136.

[32] Kılıç, K., Boyacı, İ. H., Köksel, H., and Küsmenoğlu, İ. (2007). A classification system for beans using computer vision system and artificial neural networks. Journal of Food Engineering, 78(3), 897-904.

[33] Tu, K., Ren, K., Pan, L., and Li, H. (2007). A study of broccoli grading system based on machine vision and neural networks, In: Proceedings of the IEEE International Conference on Mechatronics and Automation, August, 2007, pp. 2332-2336.

[34] Zhang, W., Bai, C., and Liu, G. (2007). Neural network modeling of ecosystems: A case study on cabbage growth system. Ecological Modelling, 201(3), 317-325.

[35] Erenturk, S., and Erenturk, K. (2007). Comparison of genetic algorithm and neural network approaches for the drying process of carrot. Journal of Food Engineering, 78(3), 905-912.

[36] Izadifar, M., and Abdolahi, F. (2006). Comparison between neural network and mathematical modeling of supercritical CO< sub $>2</$ sub $>$ extraction of black pepper essential oil. The Journal of Supercritical Fluids, 38(1) 37-43.

[37] Melin, P., Mancilla, A., Lopez, M., Trujillo, W., Cota, J., and Gonzalez, S. (2007). Modular neural networks with fuzzy integration applied for time series forecasting. In: Analysis and Design of Intelligent Systems using Soft Computing Techniques, 217-225.

[38] Prasad, R., Pandey, A., Singh, K.P., Singh, V.P., and Mishra, R.K. (2012). Retrieval of spinach crop parameters by microwave remote sensing with back propagation artificial neural networks: A comparison of different transfer functions. Advances in Space Research, 50(3), 363-370. 
[39] Karadurmus, E., Cesmeci, M., Yuceer, M., and Berber, R. (2012). An artificial neural network model for the effects of chicken manure on ground water. Applied Soft Computing, 12(1), 494-497.

[40] Hosseini, H. G., Luo, D., Xu, G., Liu, H., and Benjamin, D. (2008). Intelligent fish freshness assessment. Journal of Sensors. (Article ID 628585). doi:10.1155/2008/628585.

[41] Savegnago, R. P., Nunes, B. N., Caetano, S. L., Ferraudo, A. S., Schmidt, G. S., Ledur, M. C., and Munari, D. P. (2011). Comparison of logistic and neural network models to fit to the egg production curve of White Leghorn hens. Poultry Science, 90(3), $705-711$.

[42] Peiyuan, G., and Man, B. (2011). Pattern recognition to lamb freshness based on much data merging. In: IEEE International Conference on Mechatronics and Automation (ICMA), August 2011, pp. 1583-1587.

[43] Valous, N. A., Mendoza, F., Sun, D., and Allen, P. (2010). Supervised neural network classification of pre-sliced cooked pork ham images using quaternionic singular values. Meat Science, 84(3), 422-430.

[44] Mittal, G. S., and Zhang, J. (2000). Prediction of temperature and moisture content of frankfurters during thermal processing using neural network. Meat Science, 55(1), 13-24.

[45] Mottaghitalab, M., Faridi, A., Darmani-Kuhi, H., France, J., and Ahmadi, H. (2010). Predicting caloric and feed efficiency in turkeys using the group method of data handling-type neural networks. Poultry Science, 89(6), 1325-1331. 